\title{
An Updated Meta-Analysis: Risk Conferred by Glutathione S-Transferases (GSTM1 and GSTT1) Polymorphisms to Age-Related Cataract
}

\author{
Rong-feng Liao, ${ }^{1,2}$ Min-jie Ye, ${ }^{2}$ Cai-yuan Liu, ${ }^{3}$ and Dong-qing Ye \\ ${ }^{1}$ Department of Epidemiology and Biostatistics, School of Public Health, Anhui Medical University, 81 Meishan Road, \\ Hefei, Anhui 230032, China \\ ${ }^{2}$ Department of Ophthalmology, The First Affiliated Hospital of Anhui Medical University, Hefei, 218 Jixi Road, \\ Hefei, Anhui 230022, China \\ ${ }^{3}$ Department of Ophthalmology, The Hospital of University of Science and Technology of China, Jinzhai Road, \\ Hefei, Anhui 230026, China \\ Correspondence should be addressed to Rong-feng Liao; ophthalmology@126.com and Dong-qing Ye; ydqayd@126.com
}

Received 15 September 2014; Accepted 21 November 2014

Academic Editor: James F. Hejtmancik

Copyright (c) 2015 Rong-feng Liao et al. This is an open access article distributed under the Creative Commons Attribution License, which permits unrestricted use, distribution, and reproduction in any medium, provided the original work is properly cited.

Purpose. To study the effects of glutathione S-transferase M1 (GSTM1) and T1 (GSTT1) polymorphisms on age-related cataract (ARC). Methods. After a systematic literature search, all relevant studies evaluating the association between GSTs polymorphisms and ARC were included. Results. Fifteen studies on GSTM1 and nine studies on GSTT1 were included in this meta-analysis. In the pooled analysis, a significant association between null genotype of GSTT1 and ARC was found (OR $=1.229,95 \% \mathrm{CI}=1.057-1.429$, and $P=0.007)$. In subgroup analysis, the association between cortical cataract (CC) and GSTM1 null genotype was statistically significant $(\mathrm{OR}=0.713,95 \% \mathrm{CI}=0.598-0.850$, and $P<0.001)$. In addition, GSTM1 null genotype was significantly associated with ARC causing risk to individuals working indoors and not individuals working outdoors. The association between GSTT1 null genotype and risk of ARC was statistically significant in Asians $(\mathrm{OR}=1.442,95 \% \mathrm{CI}=1.137-1.830$, and $P=0.003)$ but not in Caucasians. Conclusions. GSTM1 positive genotype is associated with increased risk of CC and loses the protective role in persons who work outdoors. Considering the ethnic variation, GSTT1 null genotype is found to be associated with increased risk of ARC in Asians but not in Caucasians.

\section{Introduction}

Cataract is one of the most common causes of visual impairment and blindness all over the world. $80 \%$ of cataract is age-related cataract (ARC), which is classified as cortical cataract (CC), nuclear cataract (NC), or posterior subcapsular cataract (PSC), according to the location of the opacity in the lens $[1,2]$. Although the pathogenesis of ARC is not fully understood, many epidemiologic studies have noted that oxidative stress and genetic factors may play major roles in the development of ARC [3].

There are many cellular defense mechanisms that protect the human lens from oxidative damage. The glutathione S-transferase (GST) is one of the detoxification enzyme systems and plays important role in inactivating endogenous and exogenous toxic products under oxidative stress. The GST isoenzymes have been reported to express classes, mu, theta, and pi, in human lens tissue [4-7]. GSTM1/T1 polymorphisms are the most common polymorphisms of GST enzymes, and they have been associated with many diseases, such as rheumatoid arthritis, age-related macular degeneration, oral leukoplakia, prostate cancer, lung cancer, and cervical neoplasia [8-13].

Possible association between the GSTM1/T1 polymorphisms and ARC has been investigated in many studies however with conflicting results. Recently, a meta-analysis was performed to evaluate the association between GSTM1/T1 polymorphisms and ARC [14]. Ever since, new studies of 


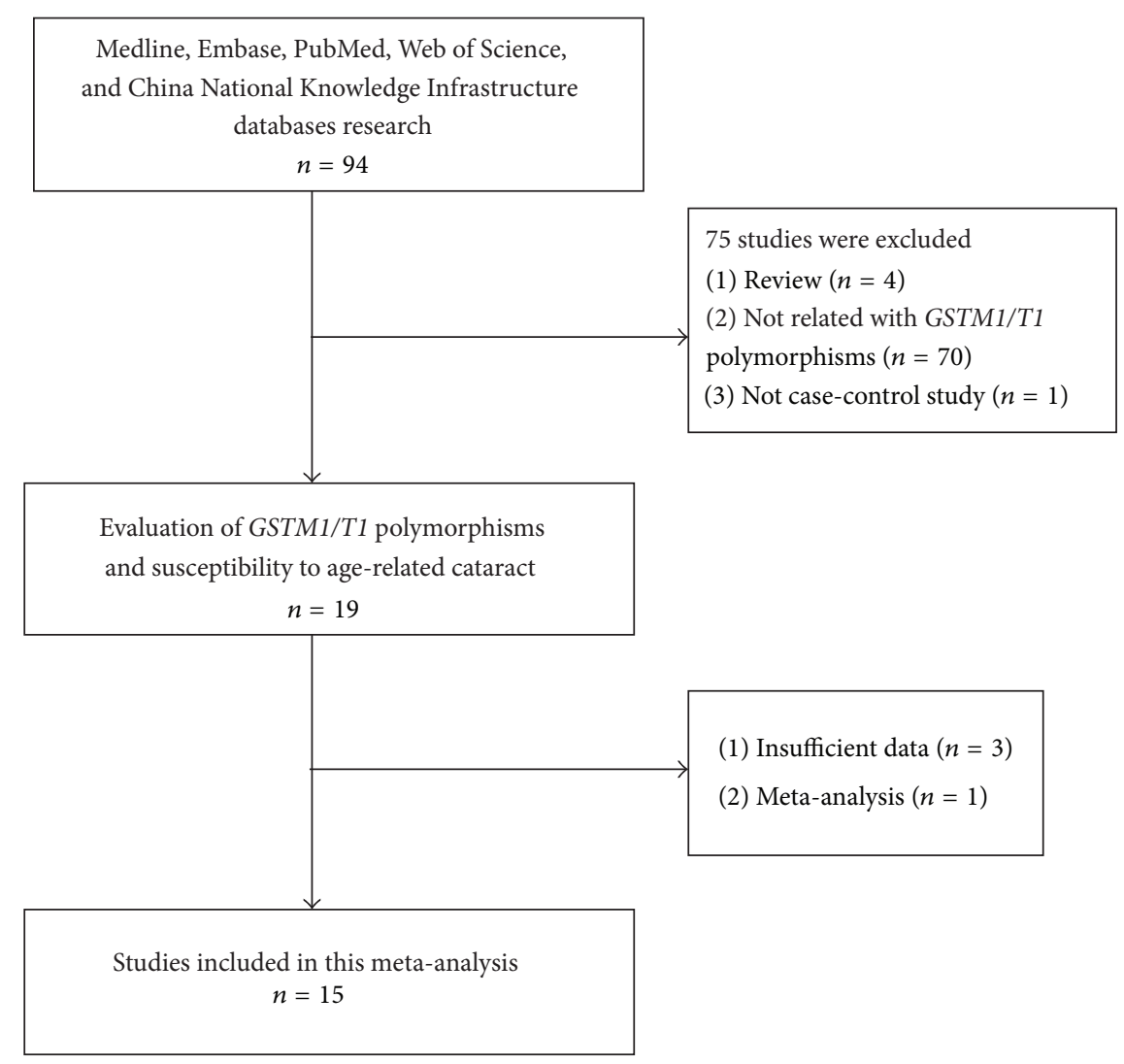

FIGURE 1: Flow chart showing study selection procedure.

GSTM1/T1 polymorphisms in cataract have been published, shedding new light on the topic. We performed an updated meta-analysis of the available studies to better ascertain the association of GSTM1/T1 polymorphisms and the risk of ARC.

\section{Materials and Methods}

2.1. Literature Search Strategy. We conducted a comprehensive systematic search to identify relevant studies from Medline, Embase, PubMed, Web of Science, and China National Knowledge Infrastructure using keyword combinations: "glutathione S-transferase or GST or GSTM1 or GSTT1" and "cataract or age-related cataract or senile cataract or ARC." When there was more than one publication using the same patient sample, only the one with the largest sample size was selected.

2.2. Inclusion Criteria and Data Extraction. Studies that met all the following criteria were regarded eligible: (1) casecontrol study, (2) investigation of the association between GSTM1/T1 polymorphisms and ARC, and (3) providing the information on genotype frequencies of GSTM1/T1 polymorphism in both cases and controls. We collected the following information from each study as initial data: the first author's name, publication year, ethnicity (country), and the number of GSTM1 and GSTT1 genotypes in the cases and controls. The articles were reviewed independently by two investigators (Minjie Ye and Caiyuan Liu), who also extracted data. The quality of studies was also evaluated based on the STROBE quality score systems [15]. A third reviewer (Rongfeng Liao) served as the third reviewer if there was any disagreement.

2.3. Statistical Analysis. The association between GSTM1/T1 polymorphisms and risk of ARC was expressed as odds ratio (OR) and 95\% confidence interval (CI). The statistical analysis was performed using Stata 11.0 (StataCorp, College Station, TX). An $\mathrm{I}^{2}$ statistic was conducted to evaluate whether inconsistencies among studies were attributed to heterogeneity rather than chance. When there was no heterogeneity of the results of the publications, we used the fixed effects model (Mantel-Haenszel method) [16]. Otherwise, we used the random effects model (DerSimonian-Laird method) [17]. Subgroup analyses were performed on the basis of ethnicity, gender, ARC subtypes, and the work place of the study subjects (indoor and outdoor work place). Finally, the Egger weighted regression method and funnel plots were used to evaluate publication bias visually.

\section{Results}

3.1. Characteristics of Studies. Flow diagram of studies included in this meta-analysis is provided in Figure 1. Fifteen studies [18-32] were included in the meta-analysis of the 
TABLE 1: Characteristics of studies included in the meta-analysis.

\begin{tabular}{|c|c|c|c|c|c|c|c|c|}
\hline \multirow{2}{*}{ First author } & \multirow{2}{*}{ Year } & \multirow{2}{*}{ Ethnicity (country) } & \multicolumn{2}{|c|}{ Sample size } & \multicolumn{2}{|c|}{ Number of null genotypes } & \multirow{2}{*}{ References } & \multirow{2}{*}{ Quality score } \\
\hline & & & Cases & Controls & Cases & Controls & & \\
\hline \multicolumn{9}{|l|}{ GSTM1: } \\
\hline $\mathrm{Pi}$ & 1996 & Asian (China) & 59 & 112 & 41 & 57 & {$[18]$} & 18 \\
\hline Hao & 1999 & Asian (China) & 77 & 76 & 41 & 35 & {$[19]$} & 17 \\
\hline $\mathrm{Xu}$ & 2007 & Asian (China) & 120 & 118 & 81 & 60 & {$[20]$} & 19 \\
\hline Zhou & 2010 & Asian (China) & 279 & 145 & 171 & 95 & {$[21]$} & 29 \\
\hline Jiang & 2012 & Asian (China) & 422 & 312 & 176 & 173 & {$[22]$} & 31 \\
\hline Sekine & 1995 & Asian (Japan) & 138 & 62 & 101 & 30 & [23] & 20 \\
\hline Sireesha & 2012 & Caucasian (India) & 455 & 205 & 177 & 94 & {$[24]$} & 32 \\
\hline Alberti & 1996 & Caucasian (Italy) & 202 & 98 & 99 & 49 & {$[25]$} & 23 \\
\hline Juronen & 2000 & Caucasian (Estonia) & 503 & 202 & 240 & 111 & {$[26]$} & 25 \\
\hline Saadat & 2004 & Caucasian (Iran) & 150 & 150 & 90 & 58 & {$[27]$} & 25 \\
\hline Saadat & 2006 & Caucasian (Iran) & 95 & 95 & 56 & 36 & {$[28]$} & 23 \\
\hline Güven & 2007 & Caucasian (Turkey) & 195 & 136 & 105 & 58 & [29] & 29 \\
\hline Abdel Azeem & 2009 & Caucasian (Egypt) & 53 & 73 & 23 & 46 & {$[30]$} & 22 \\
\hline Saadat & 2012 & Caucasian (Iran) & 186 & 195 & 104 & 89 & {$[31]$} & 26 \\
\hline Chandra & 2014 & Caucasian (India) & 131 & 126 & 43 & 68 & {$[32]$} & 25 \\
\hline \multicolumn{9}{|l|}{ GSTT1: } \\
\hline Zhou & 2010 & Asian (China) & 279 & 145 & 146 & 60 & {$[21]$} & 29 \\
\hline Jiang & 2012 & Asian (China) & 422 & 312 & 221 & 138 & {$[22]$} & 31 \\
\hline Sireesha & 2012 & Caucasian (India) & 455 & 205 & 123 & 40 & {$[24]$} & 32 \\
\hline Juronen & 2000 & Caucasian (Estonia) & 503 & 202 & 73 & 36 & {$[26]$} & 25 \\
\hline Saadat & 2004 & Caucasian (Iran) & 150 & 150 & 49 & 46 & {$[27]$} & 25 \\
\hline Güven & 2007 & Caucasian (Turkey) & 195 & 136 & 29 & 22 & {$[29]$} & 29 \\
\hline Abdel Azeem & 2009 & Caucasian (Egypt) & 53 & 73 & 16 & 21 & {$[30]$} & 22 \\
\hline Saadat & 2012 & Caucasian (Iran) & 186 & 195 & 49 & 57 & {$[31]$} & 26 \\
\hline Chandra & 2014 & Caucasian (India) & 131 & 126 & 18 & 5 & {$[32]$} & 25 \\
\hline
\end{tabular}

GSTM1: glutathione S-transferase M1; GSTT1: glutathione S-transferase T1.

GSTM1 genotype (3165 cases, 2105 controls), and nine studies were included in the meta-analysis of GSTT1 (2374 cases, 1544 controls). For the meta-analysis of GSTM1, six studies on Asians and nine on Caucasians were included. While for the analysis of GSTT1, two studies on Asians and seven studies on Caucasians were included. The characteristics of the studies included in the meta-analysis are presented in Table 1.

3.2. Meta-Analysis Results. The forest plot of the GSTM1 and GSTT1 genotypes is shown in Figures 2(a) and 2(b), respectively. No association was detected between GSTM1 null genotype and ARC in the overall analysis $(\mathrm{OR}=1.161$, 95\% CI $=0.863-1.563$, and $P=0.324)$. The association between GSTT1 null genotype and risk of ARC was statistically significant $(\mathrm{OR}=1.229,95 \% \mathrm{CI}=1.057-1.429$, and $P=0.007)$.

Subgroup analyses on ethnicity indicated that the association between GSTM1 null genotype and risk of ARC was not significant in Asians or Caucasians (OR $=1.372,95 \%$ CI $=0.786-2.396$, and $P=0.266$; $\mathrm{OR}=1.053,95 \% \mathrm{CI}=0.726-$ 1.526 , and $P=0.785$, Figure 3(a)). The association between
GSTT1 null genotype and risk of ARC was statistically significant in Asians but not in Caucasians ( $\mathrm{OR}=1.442,95 \%$ $\mathrm{CI}=1.137-1.830$, and $P=0.003 ; \mathrm{OR}=1.113,95 \% \mathrm{CI}=$ $0.830-1.492$, and $P=0.474$, resp., Figure 3(b)). In subgroup analyses, by gender, we found that GSTM1 null genotype was not associated with ARC in female or male group $(\mathrm{OR}=1.016$, $95 \% \mathrm{CI}=0.444-2.324$, and $P=0.970 ; \mathrm{OR}=0.892,95 \% \mathrm{CI}=$ $0.582-1.365$, and $P=0.598$, resp.). Similar results were found for the association between GSTT1 null genotype and risk of ARC in Asian female or male group $(\mathrm{OR}=1.281,95 \% \mathrm{CI}=$ $0.972-1.687$, and $P=0.078$; $\mathrm{OR}=1.288,95 \% \mathrm{CI}=0.977$ 1.698 , and $P=0.073$, resp.). When analyzed by subtypes of ARC, the GSTM1 null genotype was significantly correlated with CC $(\mathrm{OR}=0.713,95 \% \mathrm{CI}=0.598-0.850$, and $P<0.001$; Figure 4(a)) but not with NC, PSC, or mixed type (MT) (OR $=0.887,95 \% \mathrm{CI}=0.685-1.148$, and $P=0.363 ; \mathrm{OR}=1.042$, $95 \% \mathrm{CI}=0.797-1.362$, and $P=0.765 ; \mathrm{OR}=0.937,95 \% \mathrm{CI}$ $=0.510-1.722$, and $P=0.834$, resp.). GSTT1 null genotype was significantly correlated with PSC (OR $=1.421,95 \%$ CI $=1.043-1.936$, and $P=0.026$; Figure $4(\mathrm{~b}))$ and marginally correlated with CC $(\mathrm{OR}=1.226,95 \% \mathrm{CI}=0.999-1.504$, and 
TABLE 2: Subgroup analysis of the association between GSTM1 and GSTT1 polymorphisms and the risk of age-related cataract.

\begin{tabular}{|c|c|c|c|c|c|}
\hline Groups & Number of studies & Statistical method & OR $(95 \% \mathrm{CI})$ & $P$ & References \\
\hline \multicolumn{6}{|l|}{ GSTM1: } \\
\hline All studies & 15 & Random & $1.161(0.863-1.563)$ & 0.324 & {$[18-32]$} \\
\hline \multicolumn{6}{|l|}{ Ethnicity: } \\
\hline Asian & 6 & Random & $1.372(0.786-2.396)$ & 0.266 & {$[18-23]$} \\
\hline Caucasian & 9 & Random & $1.053(0.726-1.526)$ & 0.158 & {$[24-32]$} \\
\hline \multicolumn{6}{|l|}{ Gender: } \\
\hline Female & 5 & Random & $1.016(0.444-2.324)$ & 0.970 & {$[22,24,27,29,30]$} \\
\hline Male & 5 & Random & $0.892(0.582-1.365)$ & 0.598 & {$[22,24,27,29,30]$} \\
\hline \multicolumn{6}{|l|}{ Subtype: } \\
\hline $\mathrm{CC}$ & 6 & Fixed & $0.713(0.598-0.850)$ & $<0.001$ & {$[21,22,24-26,29]$} \\
\hline $\mathrm{NC}$ & 5 & Fixed & $0.887(0.685-1.148)$ & 0.363 & {$[21,24-26,29]$} \\
\hline PSC & 4 & Fixed & $1.042(0.797-1.362)$ & 0.765 & {$[21,24-26,29]$} \\
\hline MT & 3 & Random & $0.937(0.510-1.722)$ & 0.834 & {$[24,26,29]$} \\
\hline \multicolumn{6}{|l|}{ Environmental risk factors: } \\
\hline Outdoor & 2 & Fixed & $1.019(0.511-2.034)$ & 0.957 & {$[28,31]$} \\
\hline Indoor & 2 & Random & $2.062(1.074-3.961)$ & 0.030 & {$[28,31]$} \\
\hline \multicolumn{6}{|l|}{ GSTT1: } \\
\hline All studies & 9 & Fixed & $1.229(1.057-1.429)$ & 0.007 & {$[21,22,24,26,27,29-32]$} \\
\hline \multicolumn{6}{|l|}{ Ethnicity: } \\
\hline Asian & 2 & Fixed & $1.442(1.137-1.830)$ & 0.003 & {$[21,22]$} \\
\hline Caucasian & 7 & Fixed & $1.113(0.830-1.492)$ & 0.474 & {$[24,26,27,29-32]$} \\
\hline \multicolumn{6}{|l|}{ Gender: } \\
\hline Female & 5 & Fixed & $1.281(0.972-1.687)$ & 0.078 & {$[22,24,27,29,30]$} \\
\hline Male & 5 & Fixed & $1.288(0.977-1.698)$ & 0.073 & {$[22,24,27,29,30]$} \\
\hline \multicolumn{6}{|l|}{ Subtype: } \\
\hline $\mathrm{CC}$ & 5 & Fixed & $1.226(0.999-1.504)$ & 0.051 & {$[21,22,24,26,29]$} \\
\hline $\mathrm{NC}$ & 4 & Random & $0.921(0.524-1.617)$ & 0.774 & {$[21,24,26,29]$} \\
\hline PSC & 4 & Fixed & $1.421(1.043-1.936)$ & 0.026 & {$[21,24,26,29]$} \\
\hline MT & 3 & Random & $1.209(0.663-2.204)$ & 0.535 & {$[24,26,29]$} \\
\hline GSTM1 null + GSTT1 null: & 6 & Fixed & $1.069(0.843-1.356)$ & 0.581 & {$[22,24,26,27,29,30]$} \\
\hline GSTM1 positive + GSTT1 positive: & 6 & Random & $1.005(0.658-1.536)$ & 0.981 & {$[22,24,26,27,29,30]$} \\
\hline GSTM1 positive + GSTT1 null: & 5 & Random & $1.281(0.840-1.954)$ & 0.250 & {$[22,24,27,29,30]$} \\
\hline
\end{tabular}

GSTM1: glutathione S-transferase M1; GSTT1: glutathione S-transferase T1. CC: cortical cataract; NC: nuclear cataract; PSC: posterior subcapsular cataract; MT: mixed type cataract. Fixed: a fixed effects model (Mantel-Haenszel method); Random: the random effects model (DerSimonian-Laird method).

$P=0.051)$. However, there were no significant associations between GSTT1 null genotype and NC or MT (OR $=0.921$, $95 \% \mathrm{CI}=0.524-1.617$, and $P=0.774 ; \mathrm{OR}=1.209,95 \% \mathrm{CI}$ $=0.663-2.204$, and $P=0.535$, resp.). In subgroup analyses, by the work place, we found that the association between GSTM1 null genotype and ARC was statistically significant in the indoor subjects but not in the outdoor subjects $(\mathrm{OR}=$ 2.062, 95\% CI $=1.074-3.961$, and $P=0.030$, Figure 5(a); OR $=1.019,95 \% \mathrm{CI}=0.511-2.034$, and $P=0.957$, Figure 5(b)).

To investigate the association between profiles of GST genotypes and the risk of ARC, we examined the association between combination of GSTM1 null and GSTT1 null genotypes and risk of ARC but failed to detect any association between them in all populations $(\mathrm{OR}=1.069,95 \% \mathrm{CI}=0.843-$ 1.356 , and $P=0.581)$. Similarly, the combination of GSTM1 positive and GSTT1 positive/GSTM1 positive and GSTT1 null genotypes was not associated with ARC risk (OR $=1.005,95 \%$
$\mathrm{CI}=0.658-1.536$, and $P=0.981 ; \mathrm{OR}=1.281,95 \% \mathrm{CI}=0.840-$ 1.954 , and $P=0.250$, resp.). The results of subgroup analyses are presented in Table 2.

3.3. Potential Publication Bias. Funnel plots and Egger's test were generated to evaluate potential publication bias for GSTM1 (Figure 6(a)) and GSTT1 (Figure 6(b)). A statistically significant publication bias was detected for GSTM1 (Egger's test, $P=0.048)$, but no publication bias was detected for GSTT1 (Egger's test, $P=0.908$ ).

\section{Discussion}

Causality of age-related cataract is considered to be multifactorial, and oxidative stress and genetic factors are considered the major factors in its development. It has been noted that GST polymorphisms act as genetic risk factor for ARC. 


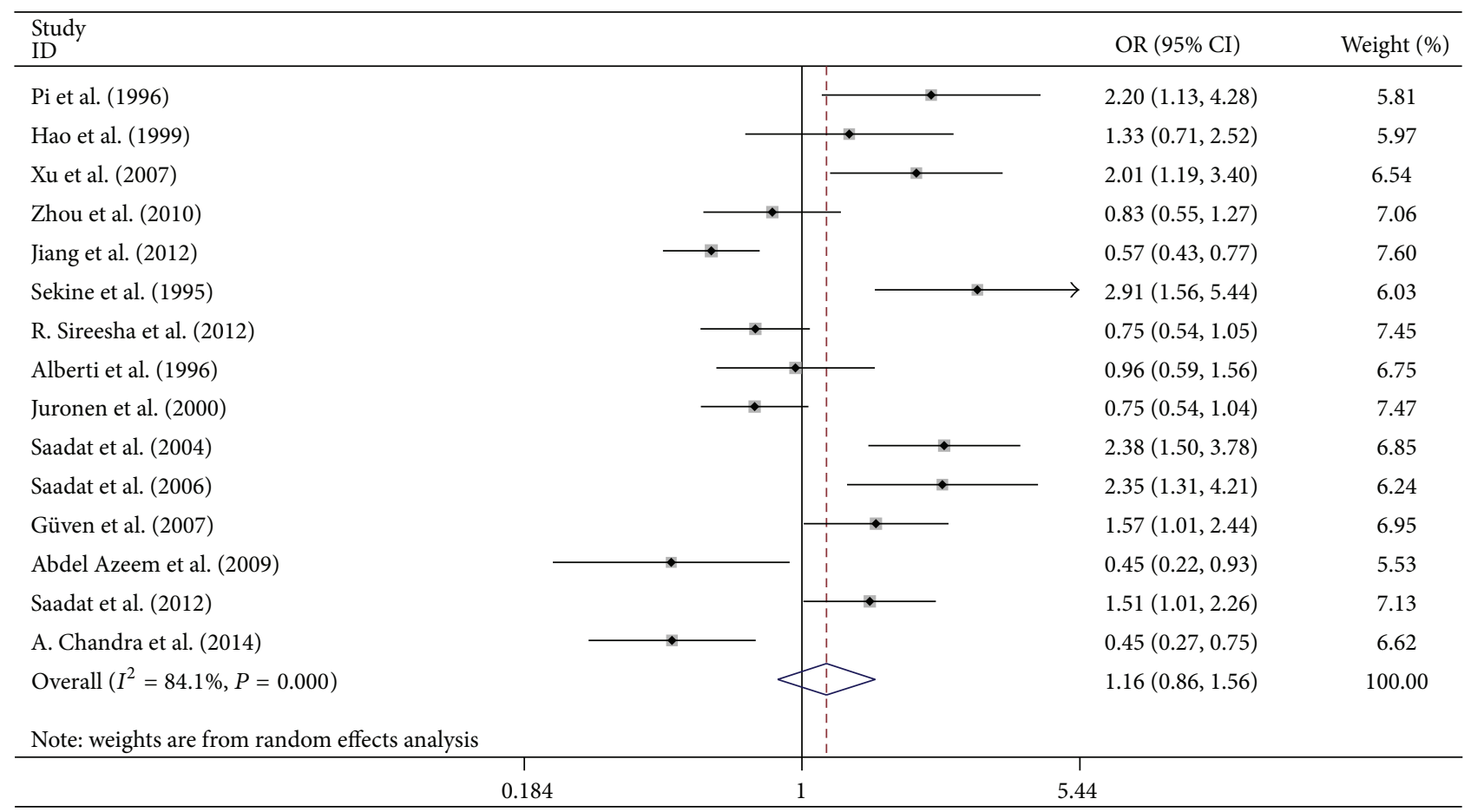

(a)

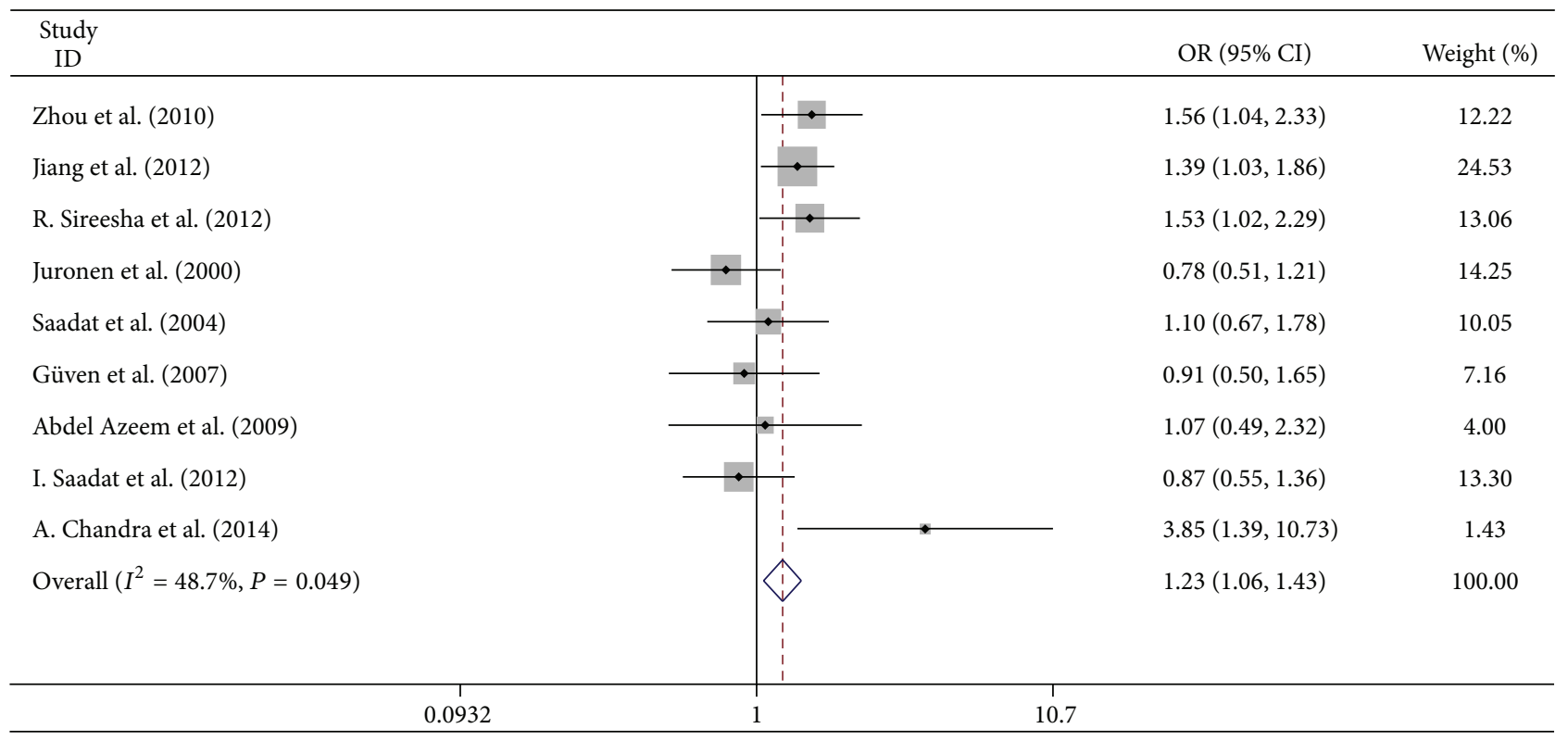

(b)

FIGURE 2: Forest plot of the association between GSTM1/T1 null genotype and age-related cataract (ARC). (a) Forest plot of the association between GSTM1 null genotype and ARC. (b) Forest plot of the association between GSTT1 null genotype and ARC.

However, results of the studies examining the association between GSTM1/T1 polymorphisms and ARC have been inconsistent. A meta-analysis performed by Sun et al. in 2010 reported that GSTM1 and GSTT1 null genotypes were associated with increased risk of ARC in Asians but not in Caucasians [14]. Thereafter, several additional clinical studies that evaluated the association of GSTM1/T1 polymorphisms and ARC have been reported. We therefore updated the present meta-analysis which included a larger sample size to provide a more reliable association between GSTM1/T1 polymorphisms and ARC susceptibility.

Compared to Sun's study, our study has some particular strength. First, we added four studies with large samples size, the absence of which might lead to a deviation in the results 


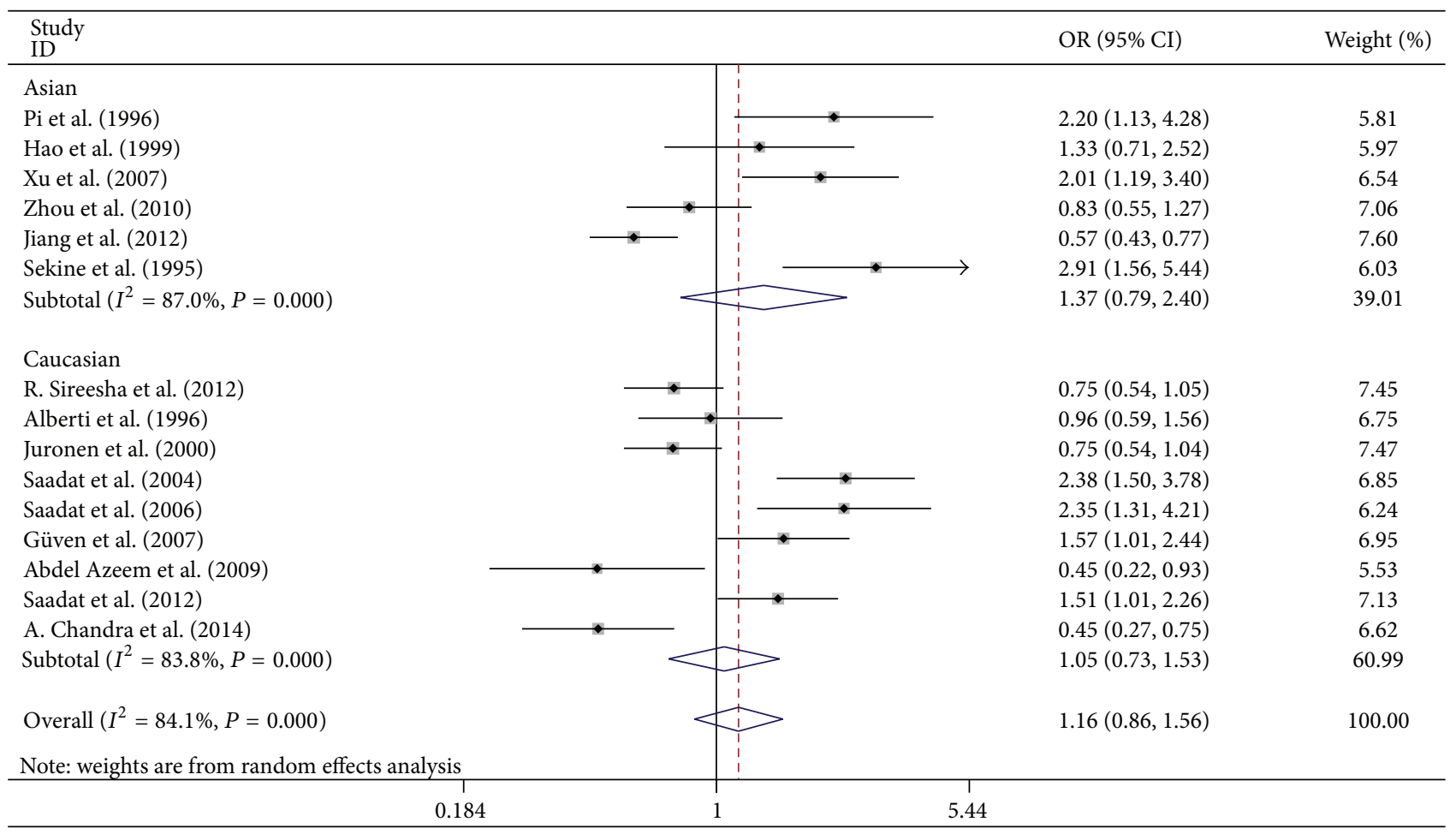

(a)

\begin{tabular}{|c|c|c|c|}
\hline $\begin{array}{l}\text { Study } \\
\text { ID }\end{array}$ & & OR $(95 \% \mathrm{CI})$ & Weight (\%) \\
\hline Asian & & & \\
\hline Zhou et al. (2010) & + & $1.56(1.04,2.33)$ & 13.60 \\
\hline Jiang et al. (2012) & $\frac{1}{1 \cdot-1}$ & $1.39(1.03,1.86)$ & 17.23 \\
\hline Subtotal $\left(I^{2}=0.0 \%, P=0.653\right)$ & & $1.44(1.14,1.83)$ & 30.83 \\
\hline \multicolumn{4}{|l|}{ Caucasian } \\
\hline R. Sireesha et al. (2012) & $\cdot$ & $1.53(1.02,2.29)$ & 13.68 \\
\hline Juronen et al. (2000) & $\longrightarrow$ & $0.78(0.51,1.21)$ & 12.67 \\
\hline Saadat et al. (2004) & $\cdot \cdot$ & $1.10(0.67,1.78)$ & 11.37 \\
\hline Güven et al. (2007) & $\longrightarrow$ i & $0.91(0.50,1.65)$ & 8.83 \\
\hline Abdel Azeem et al. (2009) & - & $1.07(0.49,2.32)$ & 6.24 \\
\hline I. Saadat et al. (2012) & $\longrightarrow \frac{1}{1}$ & $0.87(0.55,1.36)$ & 12.35 \\
\hline A. Chandra et al. (2014) & $\longrightarrow$ & $3.85(1.39,10.73)$ & 4.02 \\
\hline Subtotal $\left(I^{2}=50.7 \%, P=0.058\right)$ & & $1.11(0.83,1.49)$ & 69.17 \\
\hline Overall $\left(I^{2}=48.7 \%, P=0.049\right)$ & & $1.20(0.96,1.51)$ & 100.00 \\
\hline \multicolumn{4}{|l|}{ Note: weights are from random effects analysis } \\
\hline $\begin{array}{c}1 \\
0.0932 \\
\end{array}$ & 1 & 10.7 & \\
\hline
\end{tabular}

(b)

FIGURE 3: Ethnicity-based subgroup analysis of the correlation between GSTM1/T1 null genotype and age-related cataract (ARC). (a) Ethnicity-based subgroup analysis of the association between GSTM1 null genotype and ARC. (b) Ethnicity-based subgroup analysis of the association between GSTT1 null genotype and ARC. 


\begin{tabular}{l|llc}
\hline Study \\
ID
\end{tabular}

(a)

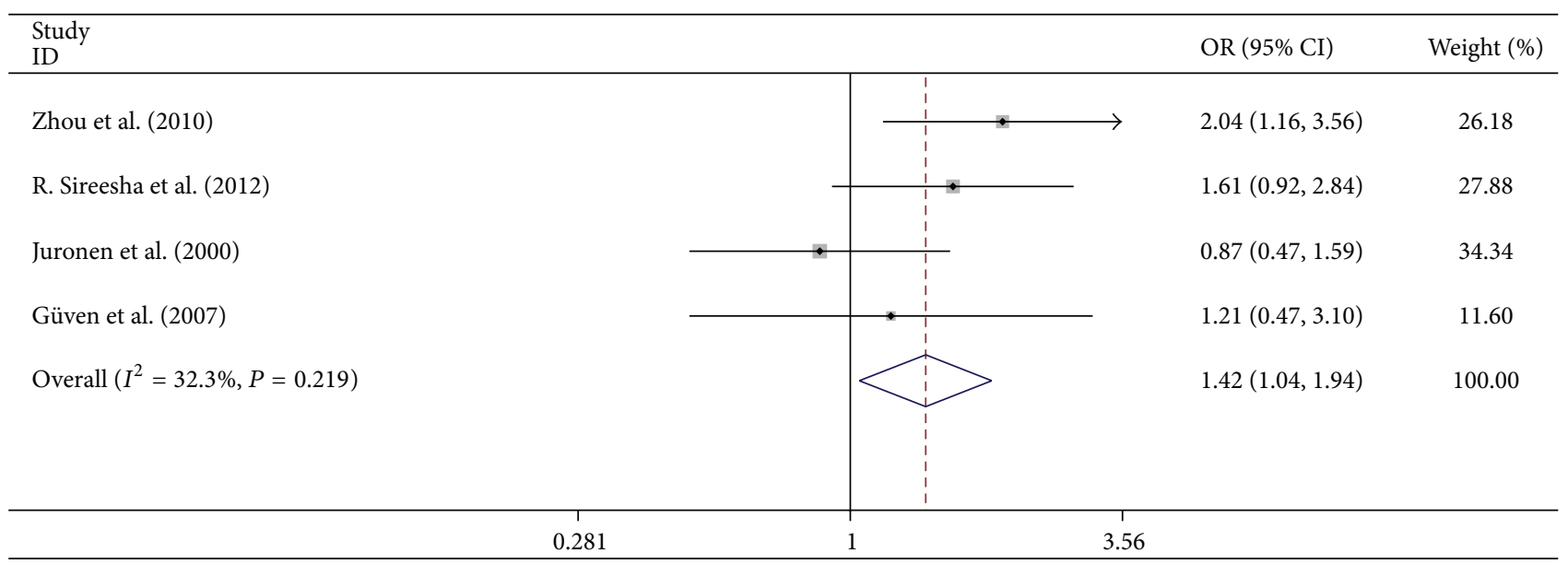

(b)

FIGURE 4: Subgroup analysis of the correlation between GSTM1/T1 null genotype and subtypes of age-related cataract (ARC). (a) Subgroup analysis of the association between GSTM1 null genotype and cortical cataract (CC). (b) Subgroup analysis of the association between GSTT1 null genotype and posterior subcapsular cataract (PSC).

of Sun's study. Second, given that GSTs play a vital role in detoxification of xenobiotics and protection of lens from the oxidative damage, we performed subgroup analysis based on the work place (outdoor/indoor) to investigate the possible contribution of GSTM1/T1 polymorphisms to susceptibility to ARC. Third, it has been noted that combination of the GST polymorphisms rather than individual polymorphism makes persons more susceptible to genotoxic insults [33]. Considering the possible additive effect of different GST genotypes, the association between the genotype profile and ARC risk was also estimated.

Inconsistent with the previous meta-analysis, our finding indicated that the GSTM1 null genotype was not associated with the ARC risk in Asian populations. Interestingly, the result of subgroup analyses based on ethnic illustrated that the GSTM1 null genotype was associated with decreased CC risk $(P<0.001)$. Meanwhile, in subgroup analysis by the work place, we found that GSTM1 positive genotype was not associated with decreased risk of ARC in outdoor subjects. Why does the GSTM1 positive genotype increase the CC risk and lose its protective role in individuals who were occupationally exposed to sunlight? The following reasons may account for the results. (1) Despite the fact that GST enzymes are important in defense against oxidative stress, they also participate in reactions that create toxic products which may result in structural alterations to the proteins and then cause lens opacification [34]. (2) The activity of GST is significantly decreased in cataractous lens compared with that in normal lens, and hence the positive genotype of GSTM1 may lose its ability to prevent cataract development. (3) Absence of the protection of GSTM1 enzyme may stimulate other cellular defense mechanisms to detoxify the substrates. (4) It has been reported that UVB (ultraviolet radiation b) irradiation results in inhibitory effect on GST activity in the skin [35]. Therefore, this might suggest that activity of GST is inhibited in the human lens after UVB irradiation. 


$\begin{aligned} & \text { Study } \\ & \text { ID }\end{aligned}$
Saadat et al. (2006)
Saadat et al. (2012)

(a)

\begin{tabular}{llll}
\hline Study \\
ID \\
\hline Saadat et al. (2006)
\end{tabular}

(b)

FIGURE 5: Working place-based subgroup analysis of the correlation between GSTM1/T1 null genotype and age-related cataract (ARC). (a) The association between GSTM1 null genotype and ARC in indoor subjects. (b) The association between GSTM1 null genotype and ARC in outdoor subjects.

In our study, GSTT1 null genotype was associated with increased risk of ARC in the Asians but not in the Caucasians, which may be due to the difference between ethnic and the distributions of GSTT1 null genotype. It has been reported that the frequency of the GSTT1 null genotype is higher in Asian population compared with other populations [36]. The frequency of GSTT1 null genotype is nearly $50 \%$ in both the Chinese and Japanese populations [37-40]. Nevertheless, the Caucasian population has a lower frequency $(11.0 \%-37.9 \%)$ $[41,42]$. Thus, different ethnic populations may have different susceptibility to ARC depending on the pattern of GSTT1 gene polymorphism. This could partly explain why the GSTT1 null genotype is associated with increased risk of ARC in the Asian population. In subgroup analyses stratified by subtypes of ARC, we also found that the GSTT1 null genotype increased the risk of CC and PSC.

Considering the role of GSTM1 positive and GSTT1 null genotype in ARC development, investigation of the association between the combination of GSTM1 positive and GSTT1 null genotypes and ARC risk should be suggested. Therefore, we did this analysis and found the combination of GSTM1 and GSTT1 null genotypes was associated with 1.281fold increased risk of ARC, although the association was not significant.
Gender differences have also been observed on the association between GST polymorphisms and human skin and colon mucosa $[43,44]$. Thus, we performed subgroup analysis on the basis of gender. Consistent with Sun's findings, our results illustrated that gender had no effect on the association between GSTM1/T1 polymorphisms and ARC.

Despite the fact that we made an accurate and comprehensive analysis, limitations still existed in our study. First, our meta-analysis only included studies with accessible fulltext articles, in English or Chinese. Therefore, the absence of some otherwise eligible studies that were unpublished or reported in other languages could lead to some inevitable publication bias. Second, due to the lack of detailed data, subgroup analysis stratified by habits like smoking and alcohol consumption was not conducted. Third, the type and degree of opacification were classified using the lens opacities classification system II (LOCS II) or lens opacities classification system III (LOCS III). Difference of classified methods among the studies might have affected the results.

In summary, this study suggested that GSTM1 positive genotype is associated with increased risk of CC and loses the protective role in persons who work outdoors. GSTT1 null genotype confers increased risk of ARC in Asians but not in Caucasians. 


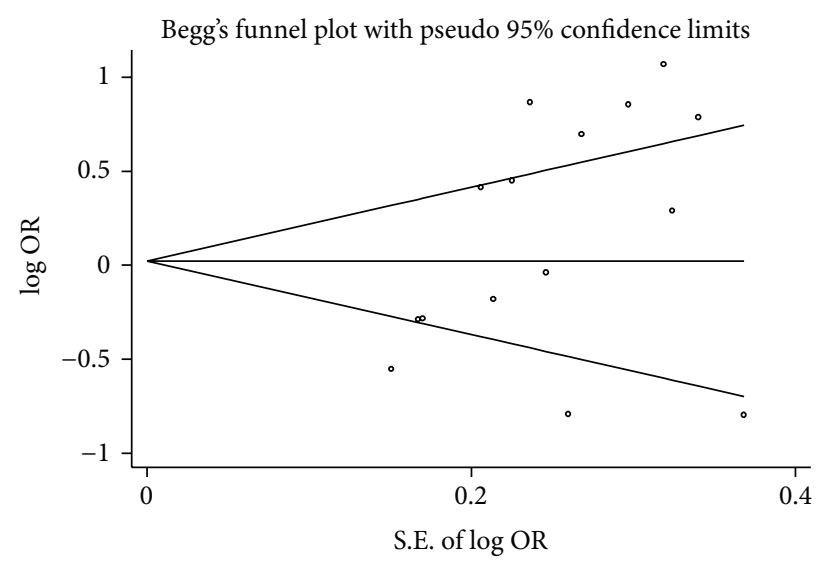

(a)

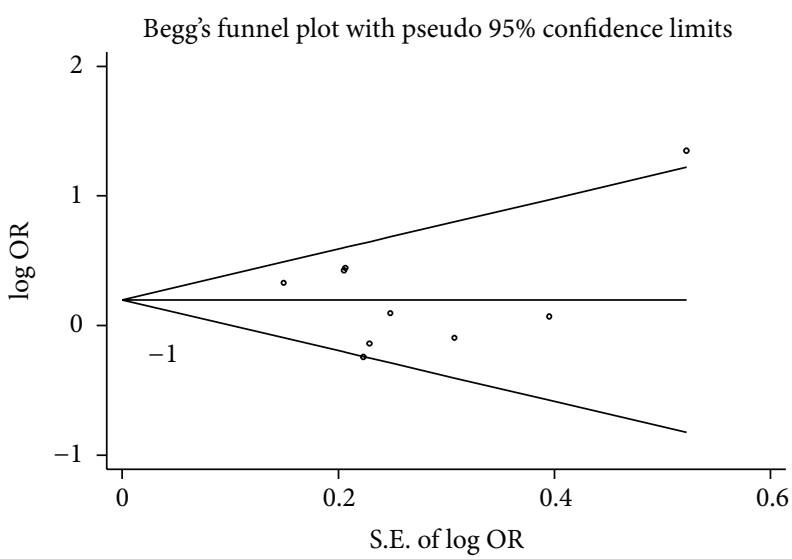

(b)

FIGURE 6: Begg's funnel plots of publication bias analyses. (a) Funnel plot of GSTM1 polymorphism and risk of ARC. (b) Funnel plot of GSTT1 polymorphism and risk of ARC.

\section{Conflict of Interests}

The authors declare no conflict of interests regarding the publication of this paper.

\section{Acknowledgments}

The authors thank Jingjing Tong and Dazhi Fan for helpful comments.

\section{References}

[1] G. Brian and H. Taylor, "Cataract blindness-challenges for the 21st century," Bulletin of the World Health Organization, vol. 79, no. 3, pp. 249-256, 2001.

[2] B. E. K. Klein, R. Klein, and K. L. P. Linton, "Prevalence of age-related lens opacities in a population: the Beaver Dam Eye Study," Ophthalmology, vol. 99, no. 4, pp. 546-552, 1992.

[3] P. A. Asbell, I. Dualan, J. Mindel, D. Brocks, M. Ahmad, and S. Epstein, "Age-related cataract," The Lancet, vol. 365, no. 9459, pp. 599-609, 2005.

[4] R. C. Strange, M. A. Spiteri, S. Ramachandran, and A. A. Fryer, "Glutathione-S-transferase family of enzymes," Mutation Research, vol. 482, no. 1-2, pp. 21-26, 2001.

[5] P. G. Board, R. T. Baker, G. Chelvanayagam, and L. S. Jermiin, "Zeta, a novel class of glutathione transferases in a range of species from plants to humans," Biochemical Journal, vol. 328, part 3, pp. 929-935, 1997.

[6] S. Marsili, R. I. Salganik, C. D. Albright et al., "Cataract formation in a strain of rats selected for high oxidative stress," Experimental Eye Research, vol. 79, no. 5, pp. 595-612, 2004.

[7] M. Ünal, M. Güven, K. Devranoğlu et al., "Glutathione S transferase $\mathrm{M} 1$ and $\mathrm{T} 1$ genetic polymorphisms are related to the risk of primary open-angle glaucoma: a study in a Turkish population," British Journal of Ophthalmology, vol. 91, no. 4, pp. 527530, 2007.

[8] J. D. Ji and W. J. Lee, "Association between the polymorphisms of glutathione S-transferase genes and rheumatoid arthritis: a meta-analysis," Gene, vol. 521, no. 1, pp. 155-159, 2013.
[9] M. Güven, E. Görgün, M. Ünal et al., "Glutathione S-transferase M1, GSTT1 and GSTP1 genetic polymorphisms and the risk of age-related macular degeneration," Ophthalmic Research, vol. 46, no. 1, pp. 31-37, 2011.

[10] E. C. Barroso Duarte, M. S. Lopes da Silva, M. Vinícius Gomez, and R. Santiago Gomez, "GSTT1 polymorphism and oral leukoplakia," Anticancer Research, vol. 26, no. 1A, pp. 427430, 2006.

[11] M. Gong, W. Dong, Z. Shi, Y. Xu, W. Ni, and R. An, "Genetic polymorphisms of GSTM1, GSTT1, and GSTP1 with prostate cancer risk: a meta-analysis of 57 studies," PLOS ONE, vol. 7, no. 11, Article ID e50587, 2012.

[12] L.-B. Gao, X.-M. Pan, L.-J. Li et al., "Null genotypes of GSTM1 and GSTT1 contribute to risk of cervical neoplasia: an evidencebased meta-analysis," PLoS ONE, vol. 6, no. 5, Article ID e20157, 2011.

[13] K. Liu, X. Lin, Q. Zhou et al., "The associations between two vital GSTs genetic polymorphisms and lung cancer risk in the Chinese population: evidence from 71 studies," PLoS ONE, vol. 9, no. 7, Article ID e102372, 2014.

[14] L. Sun, B. Xi, L. Yu et al., "Association of glutathione S-transferases polymorphisms (GSTM1 and GSTT1) with senile cataract: a meta-analysis," Investigative Ophthalmology and Visual Science, vol. 51, no. 12, pp. 6381-6386, 2010.

[15] J. P. Vandenbroucke, E. von Elm, D. G. Altman et al., "Strengthening the reporting of observational studies in epidemiology (STROBE): explanation and elaboration," Epidemiology, vol. 18, no. 6, pp. 805-835, 2007.

[16] N. Mantel and W. Haenszel, "Statistical aspects of the analysis of data from retrospective studies of disease," Journal of the National Cancer Institute, vol. 22, no. 4, pp. 719-748, 1959.

[17] R. DerSimonian and N. Laird, "Meta-analysis in clinical trials," Controlled Clinical Trials, vol. 7, no. 3, pp. 177-188, 1986.

[18] J. Pi, Y. Bai, and Q. Zheng, "A study on relationship between glutathione S-transferase mu gene deletion and senile cataract susceptibility," Zhonghua Yan Ke Za Zhi, vol. 32, no. 3, pp. 224-226, 1996 (Chinese).

[19] Y. Hao, S. He, Z. Gu et al., "Relationship between GSTM1 genotype and susceptibility to senile cataract," Zhonghua Yan Ke Za Zhi, vol. 35, no. 2, pp. 104-106, 1999 (Chinese). 
[20] M. F. Xu, S. H. Fu, and L. H. Zhang, "Relationship between GSTM1 gene deletion and susceptibility to senile cataract," Acta Academiae Medicinae Jiangxi, vol. 47, no. 4, pp. 33-38, 2007 (Chinese).

[21] J. Zhou, J. Hu, and H. Guan, "The association between copy number variations in glutathione S-transferase M1 and T1 and age-related cataract in a Han Chinese population," Investigative Ophthalmology and Visual Science, vol. 51, no. 8, pp. 3924-3928, 2010.

[22] Z. Jiang, K. Liang, Q. Zhang, and L. Tao, "Glutathione S-transferases polymorphisms confer susceptibility to senile cortical cataract in the Han Chinese population," Molecular Vision, vol. 18, pp. 1247-1252, 2012.

[23] Y. Sekine, S. Hommura, and S. Harada, "Frequency of glutathione-S-transferase 1 gene deletion and its possible correlation with cataract formation," Experimental Eye Research, vol. 60, no. 2, pp. 159-163, 1995.

[24] R. Sireesha, S. G. B. Laxmi, M. Mamata et al., "Total activity of glutathione-S-transferase (GST) and polymorphisms of GSTM1 and GSTT1 genes conferring risk for the development of age related cataracts," Experimental Eye Research, vol. 98, no. 1, pp. 67-74, 2012.

[25] G. Alberti, M. Oguni, M. Podgar et al., "Glutathione S-transferase M1 genotype and age-related cataracts: lack of association in an Italian population," Investigative Ophthalmology \& Visual Science, vol. 37, no. 6, pp. 1167-1173, 1996.

[26] E. Juronen, G. Tasa, S. Veromann et al., "Polymorphic glutathione $S$-transferases as genetic risk factors for senile cortical cataract in Estonians," Investigative Ophthalmology and Visual Science, vol. 41, no. 8, pp. 2262-2267, 2000.

[27] M. Saadat, M. Farvardin-Jahromi, and H. Saadat, "Null genotype of glutathione S-transferase M1 is associated with senile cataract susceptibility in non-smoker females," Biochemical and Biophysical Research Communications, vol. 319, no. 4, pp. 12871291, 2004.

[28] M. Saadat and M. Farvardin-Jahromi, “Occupational sunlight exposure, polymorphism of glutathione S-transferase M1, and senile cataract risk," Occupational and Environmental Medicine, vol. 63, no. 7, pp. 503-504, 2006.

[29] M. Güven, M. Ünal, A. Sarici, A. Özaydin, B. Batar, and K. Devranoğlu, "Glutathione-S-transferase M1 and T1 genetic polymorphisms and the risk of cataract development: a study in the Turkish population," Current Eye Research, vol. 32, no. 5, pp. 447-454, 2007.

[30] A. A. Abdel Azeem, A. A. Mahmoud, M. M. Salaheldine, and K. Amr, "Implication of glutathione S-transferase $\mathrm{Ml}$ and $\mathrm{Tl}$ polymorphisms in the development of senile cataract among Egyptians," Bratislava Medical Journal, vol. 110, no. 11, pp. 678-683, 2009.

[31] I. Saadat, Z. Ahmadi, M. Farvardin-Jahromi, and M. Saadat, "Association between cataract and genetic polymorphisms of GSTM1, GSTT1, and GSTO2 with respect of work place," Molecular Vision, vol. 18, pp. 1996-2000, 2012.

[32] A. Chandra, S. T. Raza, and S. Abbas, "Polymorphism of GST and FTO genes in risk prediction of cataract among a north Indian population," Ophthalmic Genetics, 2014.

[33] K. Tars, A.-K. Larsson, A. Shokeer, B. Olin, B. Mannervik, and G. J. Kleywegt, "Structural basis of the suppressed catalytic activity of wild-type human glutathione transferase T1-1 compared to its W234R mutant," Journal of Molecular Biology, vol. 355, no. 1, pp. 96-105, 2006.
[34] E. Juronen, G. Tasa, S. Veromann et al., "Polymorphic glutathione S-transferase M1 is a risk factor of primary open-angle glaucoma among Estonians," Experimental Eye Research, vol. 71, no. 5, pp. 447-452, 2000.

[35] K. I. Seo, K. H. Cho, K. C. Park et al., "Change of glutathione S-transferases in the skin by ultraviolet B irradiation," Journal of Dermatological Science, vol. 13, no. 2, pp. 153-160, 1996.

[36] A. M. Moyer, O. E. Salavaggione, S. J. Hebbring et al., "Glutathione S-transferase $\mathrm{T} 1$ and $\mathrm{M} 1$ : gene sequence variation and functional genomics," Clinical Cancer Research, vol. 13, no. 23, pp. 7207-7216, 2007.

[37] G. Wang, L. Zhang, and Q. Li, "Genetic polymorphisms of GSTT1, GSTM1, and NQO1 genes and diabetes mellitus risk in Chinese population," Biochemical and Biophysical Research Communications, vol. 341, no. 2, pp. 310-313, 2006.

[38] S.-L. Zhong, S. Zhou, X. Chen, and M. Huang, "Rapid determination of common mutations in glutathione S-transferase gene by PCR-based methods in healthy Chinese," Clinica Chimica Acta, vol. 364, no. 1-2, pp. 205-208, 2006.

[39] M. Ueda, Y.-C. Hung, Y. Terai et al., "Glutathione-S-transferase and p53 polymorphisms in cervical carcinogenesis," Gynecologic Oncology, vol. 96, no. 3, pp. 736-740, 2005.

[40] T. Katoh, H. Inatomi, H. Kim, M. Yang, T. Matsumoto, and T. Kawamoto, "Effects of glutathione S-transferase (GST) M1 and GSTT1 genotypes on urothelial cancer risk," Cancer Letters, vol. 132, no. 1-2, pp. 147-152, 1998.

[41] C. Steinhoff, K. H. Franke, K. Golka et al., "Glutathione transferase isozyme genotypes in patients with prostate and bladder carcinoma," Archives of Toxicology, vol. 74, no. 9, pp. 521-526, 2000.

[42] C. Marinho, I. Alho, D. Arduíno, L. M. Falcão, J. Brás-Nogueira, and M. Bicho, "GST M1/T1 and MTHFR polymorphisms as risk factors for hypertension," Biochemical and Biophysical Research Communications, vol. 353, no. 2, pp. 344-350, 2007.

[43] S. S. Singhal, M. Saxena, S. Awasthi et al., "Glutathione S-transferases of human skin: qualitative and quantitative differences in men and women," Biochimica et Biophysica Acta: Protein Structure and Molecular Enzymology, vol. 1163, no. 3, pp. 266272, 1993.

[44] H. Hoensch, W. H. M. Peters, H. M. J. Roelofs, and W. Kirch, "Expression of the glutathione enzyme system of human colon mucosa by localisation, gender and age," Current Medical Research and Opinion, vol. 22, no. 6, pp. 1075-1083, 2006. 


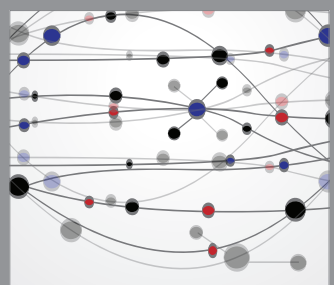

The Scientific World Journal
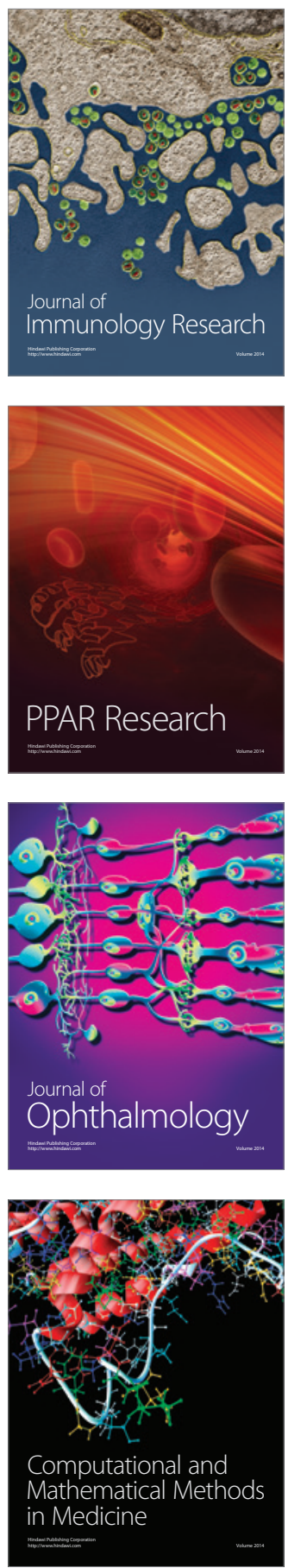

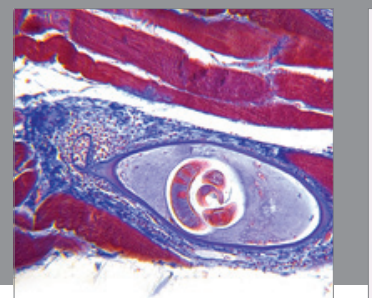

Gastroenterology

Research and Practice
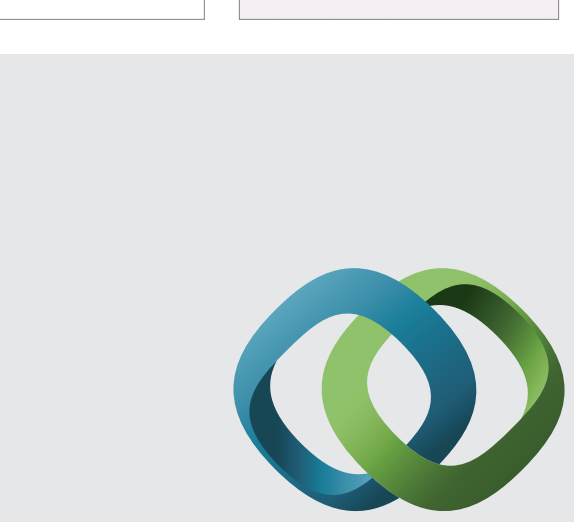

\section{Hindawi}

Submit your manuscripts at

http://www.hindawi.com
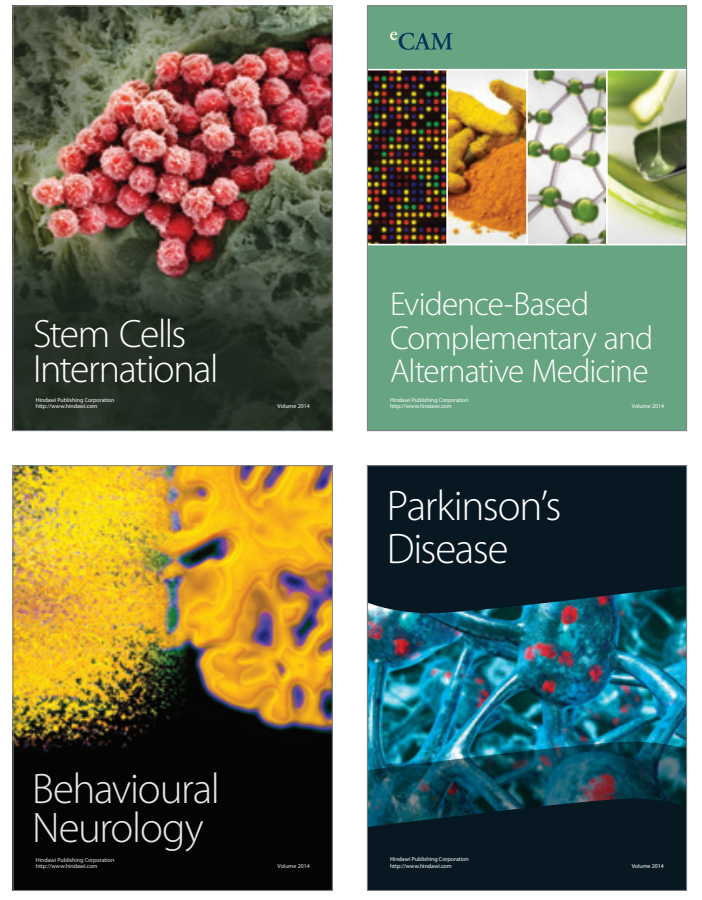
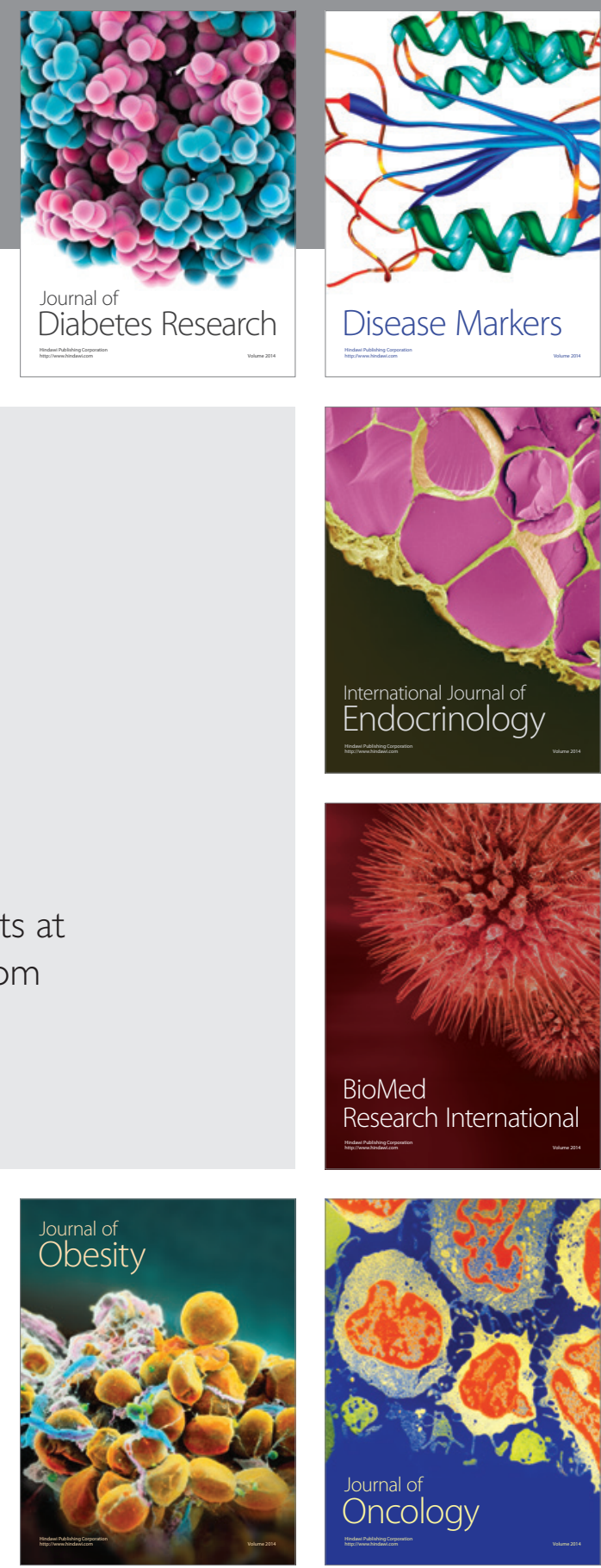

Disease Markers
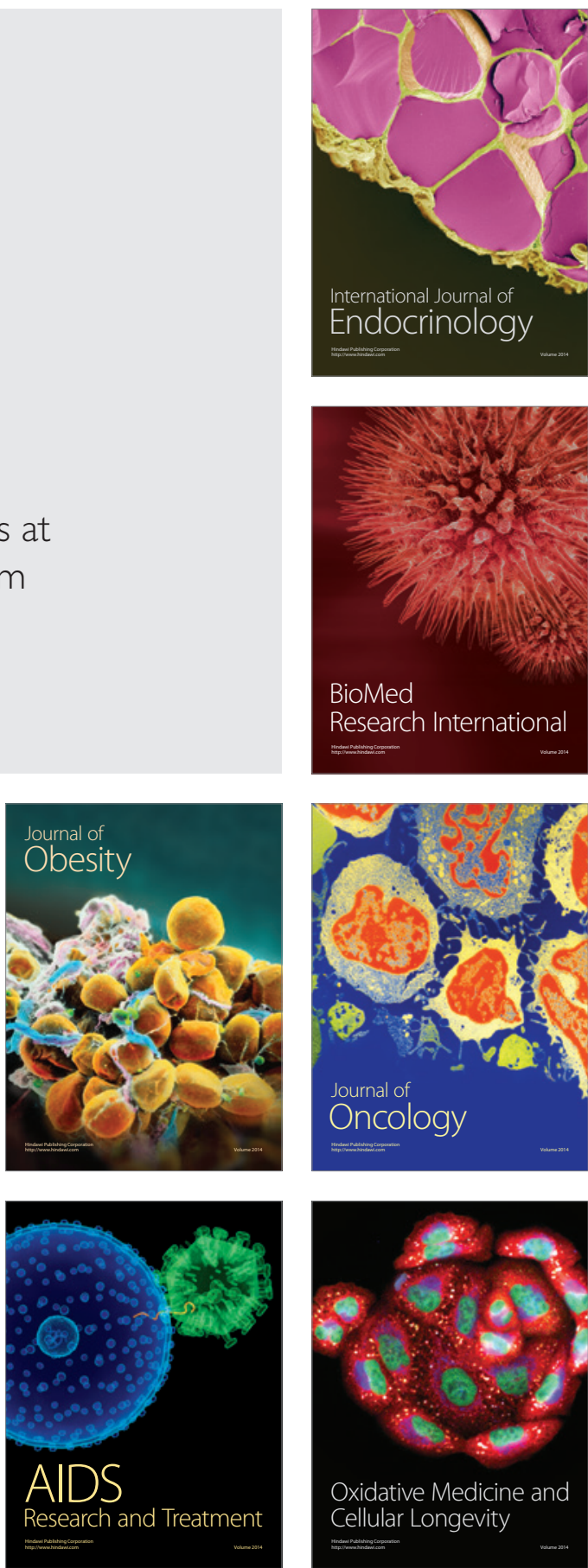